The Astrophysical Journal Supplement Series, 90:775-782, 1994 February

(c) 1994. The American Astronomical Society. All rights reserved. Printed in U.S.A.

\title{
EVIDENCE FOR PARTICLE ACCELERATION IN A MAGNETIZED WHITE DWARF FROM RADIO AND GAMMA-RAY OBSERVATIONS
}

\author{
O. C. DE JAGER \\ Department of Physics, PU for CHE, Potchefstroom 2520, South Africa \\ Received 1993 March 8; accepted 1993 July 21
}

\begin{abstract}
The DQ Her-type magnetic cataclysmic variable AE Aqr contains the most rapidly spinning white dwarf $(P=$ $33 \mathrm{~s}$ ) in a close binary and can be considered as the "millisecond pulsar" equivalent of white dwarfs. It shows flare-like UV emission on timescales of an hour during which strong QPO activity is seen, as well as flare-like radio synchrotron emission on similar timescales up to frequencies of at least $250 \mathrm{GHz}$. It is one of the most efficient converters of accretion power to $\mathrm{MeV}$ electrons of all X-ray binaries, LMXB, and cataclysmic variables. $\mathrm{TeV}$ emission was also reported by two independent groups with a periodic and burst-like behavior similar to that seen in optical. The detection of a period derivative near $10^{-13} \mathrm{~s} / \mathrm{s}$ in optical implies that the white dwarf is spinning down at a rate of $\sim 6 \times 10^{33} \mathrm{ergs} \mathrm{s}^{-1}$ which is at least an order of magnitude larger than the quiescent accretion luminosity and a few times larger than the typical UV flare luminosities. This spindown energy is not seen as disk luminosity, and the conditions appear to be favorable for the release of relativistic particles in a pulsar-type mechanism to explain the radio synchrotron emission in magnetic reconnection events, and the acceleration of particles to $\mathrm{TeV}$ energies in double layers if conditions are favorable.
\end{abstract}

Subject headings: acceleration of particles — binaries: close — stars: flare - stars: individual (AE Aquarii) white dwarfs

\section{INTRODUCTION}

This paper reviews the evidence of particle acceleration that has been found in an unusual accreting white dwarf, AE Aquarii, which is a member of the DQ Her-type magnetic cataclysmic variables. It is optically bright enough to be even visible to amateur astronomers, since the visual magnitude varies between 12.5 (during quiescence) up to 10 (in the flaring state.) Its distance to Earth is $d \sim 84 \mathrm{pc}$ and the quiescent accretion luminosity is $\sim 10^{32} \mathrm{ergs} \mathrm{s}^{-1}$, but increases to a few times $10^{33} \mathrm{ergs} \mathrm{s}^{-1}$ during strong flares (Van Paradijs et al. 1989). The distance may be $\sim 150$ pc (B. Warner 1993, personal communication ), which would scale the luminosity estimates correspondingly.

The total light from the system is dominated by the presence of the $\sim \mathrm{K} 4 \mathrm{~V}$ dwarf-type secondary spectrum, when the system is in quiescence, but about $30 \%$ of the total optical light is due to the accretion disk (Welsh, Horne, \& Oke 1993). The results of such infrared to ultraviolet observations are indicated as crosses on Figure 1. The data for this figure were taken from Szkody (1977), Tanzi et al. (1981), Berriman et al. (1981), and Van Paradijs et al. (1989). A simple blackbody spectrum for a K4V secondary, given a distance of $84 \mathrm{pc}$, is also indicated on the figure.

Flares occur quite regularly on a timescale of a few hours with colors that are dominant in ultraviolet (Van Paradijs et al. 1989 ), indicating an origin near the disk inner edge, or, on the surface of the white dwarf where higher temperatures are expected. A typical UV flare spectrum is also indicated on Figure 1. It is thus clear that the luminosity from accretion dominates that of the secondary star ( which has $L_{\mathrm{K} 4 \mathrm{~V}}=7 \times 10^{32} \mathrm{ergs} \mathrm{s}^{-1}$ ) only when the system flares.

The white dwarf in AE Aqr has a spin period of $33.0767 \mathrm{~s}$ which is seen in optical (Patterson 1979) with a pulsed fraction which is about $0.2 \%$ of the total intensity around $5 \times 10^{14} \mathrm{~Hz}$ (i.e., visible light). The pulsed fraction rises to $\sim 1 \%$ in the $U$ band (the oscillation spectrum measured by Welsh et al. (1993) is also shown on Fig. 1). In the far-UV the pulsed fraction becomes about $40 \%$ as measured by Eracleous \& Horne (1993, personal communication) with the HST. Welsh et al. (1993) have shown that the oscillation spectrum is consistent with a blackbody spectrum, and from Figure 1 we obtain a temperature of $\sim 43,000 \mathrm{~K}$ and an emitting radius of $\sim 2$ $\times 10^{8} \mathrm{~cm}$. Comparing the pulse timing and the absorption-line orbits, Welsh, Horne, \& Gomer (1993) have shown that the optical oscillations originate on the white dwarf and are not due to reprocessing somewhere in the disk. Thus, the optical oscillations appear to come from a small area on the white dwarf, and since the pulse profile is a double sinusoid (giving rise to a $16.5 \mathrm{~s}$ period) with one pulse stronger than the other, it seems quite likely to have the two polar caps as the origin as originally suggested by Patterson (1979). An example of the power spectrum taken in visible light is shown in Figure 2 during quiescence (Fig. $2 a$ shows the spin frequency $F_{0}$ and its first overtone at $2 F_{0}$.) and in the flaring state (Fig. $2 b$ ) where QPO structures appear near $F_{0}$ and $2 F_{0}$. Notice the remarkable coherent structure which is slightly redshifted relative to $F_{0}$. This unique feature manifests regularly during flares and was also claimed in $\mathrm{TeV} \gamma$-rays (see $\$ 3.1$.)

The rapid rotation of AE Aqr is unusual for white dwarfs, and this source can be considered as the "millisecond pulsar" equivalent of white dwarfs, since its period is not much larger than the minimum period for a white dwarf rotating close to breakup speed:

$$
P_{\min }=2 \pi\left(\frac{R^{3}}{G M}\right)^{1 / 2}=12 M_{1}^{-1} \mathrm{~s}
$$




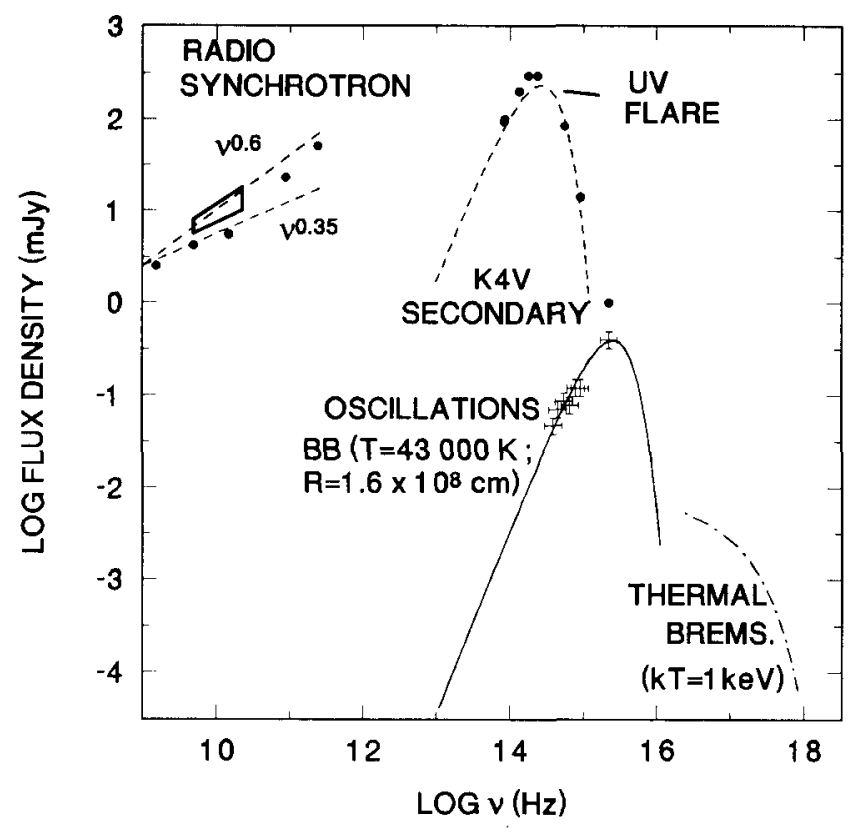

FIG. 1.- Radio to X-ray spectrum of AE Aqr. The solid circles and two dashed lines in the radio region indicate the range for the time-averaged radio spectra. The trapesium represents a range wherein many radio detections were reported (reproduced from Abada-Simon et al. 1993.) The various infrared to ultraviolet components are also indicated by solid circles ( see text). A typical visual to ultraviolet flare spectrum is shown by the short (nearly horizontal) line. A simple blackbody spectrum for a $\mathrm{K} 4 \mathrm{~V}$ dwarf star (distance $=84 \mathrm{pc}$ ) are shown by a dashed curved spectrum. The information on the blackbody (indicated as "BB") optical oscillation spectrum is described in the text. The dash-dot line indicates the observed $\mathrm{X}$-ray thermal bremsstrahlung spectrum with $k T=1 \mathrm{keV}$ (Eracleous et al. 1991).

when using the approximate mass-radius relationship of Warner \& Wickramasinghe (1991). For neutron stars this minimum period is close to $0.5 \mathrm{~ms}$, whereas the minimum observed period for pulsars is $1.5 \mathrm{~ms}$ for PSR $1937+214$. The presence of QPO features in optical (and possibly also in $\mathrm{TeV}$ $\gamma$-rays) is interesting since the accreting low-mass X-ray binaries (LMXB), which are considered to contain maximally spun-up millisecond pulsars, also exhibit QPO features, depending on the accretion rate.

In the original model for AE Aqr, Patterson (1979) have shown that this white dwarf is likely to be fed by an accretion disk which is disrupted near the corotation radius $\left(r_{i} \sim r_{c}=\right.$ $1.5 \times 10^{9} \mathrm{~cm}$ ), which is not much larger than the stellar radius (this also reminds us of the LMXB where the disk inner edge radius is considered to be near the neutron star's surface), and the two poles of the white dwarf sweeping like a lighthouse will cause the observation of a $16.5 \mathrm{~s}$ period (i.e., $2 F_{0}$ ) as well. The disruption of the inner disk is cased by the white dwarf's own magnetic field. The magnetic moment of $\sim 5 \times 10^{32} \mathrm{G} \mathrm{cm}^{3}$ follows from the observed $P_{\text {spin }}$ versus $P_{\text {orb }}$ relationship for the three known DQ Her stars (Warner \& Wickramashinghe 1991).

The system is also seen in soft X-rays with a thermal bremstrahlung temperature of $k T \sim 1 \mathrm{keV}$ (Patterson et al. 1980; Eracleous et al. 1991). This spectrum is also shown on Figure
1. The X-ray pulse profile of the $33 \mathrm{~s}$ signal is a single broad pulse with no evidence of a first harmonic component (as seen in optical ). The corresponding pulsed fraction is $~ 20 \%-30 \%$ of the total X-ray intensity (Patterson et al. 1980). Thus, the energy spectra (Fig. 1) and pulse profiles of the optical and $\mathrm{X}$-ray pulses are different, which suggests that they have different origins: the optical from the polar caps and the $\mathrm{X}$-rays most likely from that part of the stellar surface which is connected to the disk inner edge (with radius $r_{i}$ ) via the dipolar magnetic field lines. This part is also referred to as the "accretion curtain" (see, e.g., Lamb 1988).

\section{EVIDENCE FOR PARTICLE ACCELERATION FROM RADIO AND MILLIMETER OBSERVATIONS}

Radio emission from AE Aqr has been discovered by Bookbinder \& Lamb (1987). Detailed radio observations by Bastian, Dulk, \& Chanmugan (1988), (hereafter BDC) have revealed the existence of radio flares up to at least $23 \mathrm{GHz}$. It is interesting to note that the radio flares have timescales comparable to the optical flare timescales, and it was shown that the radio emission is neither of thermal origin, nor due to coherent processes.

\subsection{Optically Thick Synchrotron Radiation}

BDC have also shown that the radio data are consistent with a model of relativistic electrons radiating optically thick synchrotron emission in magnetized expanding clouds with initial magnetic field strengths of $B_{0}>25 \mathrm{G}$. In this case the relativistic electron number density spectrum is assumed to follow a power law of the form $n(E) d E=K E^{-\gamma} d E$ with losses

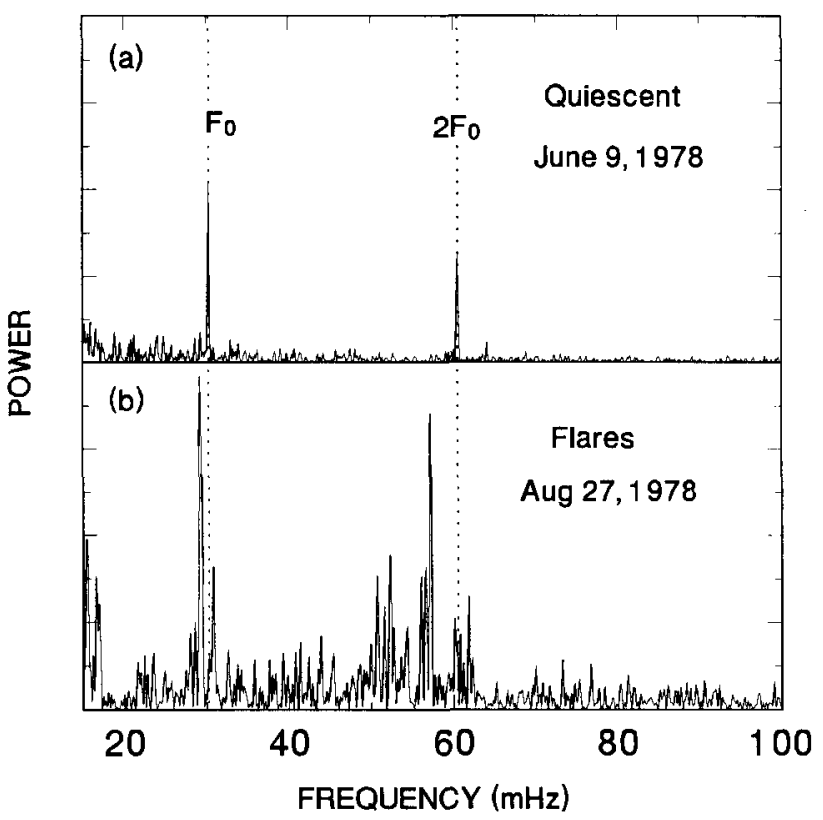

FIG. 2.-Reproduction of two Fourier spectra reported by Patterson (1979) calculated from data taken during quiescence $(a)$ and flares $(b)$. The two vertical dotted lines indicate the spin frequency $\left(F_{0}\right)$ of the white dwarf and its first overtone $\left(2 F_{0}\right)$. 
mainly confined to abiabatic losses (provided that $E$ is small enough.) As the cloud expands, $B$ also decreases. The radio emission is optically thick so that a rising spectrum is seen up to a turnover frequency of $\nu_{m}$, where the optical depth of the plasmoid is $\tau \sim 1$. At $\nu>\nu_{m}$, optically thin emission is expected. The turnover frequency increases with increasing $B_{0}$ and the detection of AE Aqr at millimeter wavelengths was predicted on the basis of this model. Such radiation was indeed confirmed at frequencies up to $250 \mathrm{GHz}$ on timescales of $\sim 15$ minutes with intensities up to $0.1 \mathrm{Jy}$, and $B_{0}$ was shown to vary between 25 and $250 \mathrm{G}$ (Abada-Simon et al. 1993.) BDC have shown that the spectral index of the time averaged radio emission depends on frequency as $\propto \nu^{\alpha}$ with $\alpha=5(2-\epsilon) / 2$ if the frequency distribution of initial flux densities $S_{0}$ is given by $f\left(S_{0}\right) \propto S_{0}^{-\epsilon}$. Abada-Simon et al. 1993 have shown that the data supports $\alpha=0.34-0.59$ implying that $\epsilon=1.76-1.86$. The radio spectrum of AE Aqr based on observations between 1984 and 1991 is shown in Figure 1.

\subsection{Optically Thin Synchrotron Radiation}

From the above-mentioned data one can estimate the total amount of energy in a flare (which amounts to at least $10^{34}$ ergs per large flare) and the field strength, but no reliable calculation of the spectral index $\gamma$ of accelerated particles can be made. However, the spectrum must turn over into optically thin synchrotron radiation at $\nu>250 \mathrm{GHz}$. This also allows us to probe the acceleration region: multicolor high-speed infrared photometry at frequencies $>3 \times 10^{13} \mathrm{~Hz}$ are scheduled for AE Aqr during 1993, which should allow the detection of the high-energy tail of the synchrotron spectrum, provided that the electron spectral index $\gamma$ is not too large. The electron energies would be required to have

$$
E>100\left(\frac{B_{0}}{250 \mathrm{G}}\right)^{-1 / 2} \mathrm{MeV}
$$

If the flares of relativistic electrons also originate in the accretion disk (as is the case of the thermal UV flares which show the disk-like QPO features as shown in Fig. $2 b$ ), we may also see QPO features associated with the synchrotron emission. The condition for this is that the electron must lose energy in a time that is less than the QPO period (so that the periodicity can be maintained). The synchrotron lifetime of such energetic electrons (lower energy electrons would lose energy mainly by adiabatic expansion ) would be $<40 \mathrm{~s}$, and if the acceleration process is periodic or quasi-periodic, we may expect the detection of -such nonthermal temporal features, which will lead to a diagnostic of the site where particle acceleration is taking place. In the infrared range the QPO must have a strong red color due to the optically thin synchrotron spectrum of the form

$$
I(\nu) \propto \nu^{-(\gamma-1) / 2} .
$$

This should lead to a measurement of the electron spectral index $\gamma(>2$ as expected). However, the thermal flares and QPO are dominant in the ultraviolet, so that it should be rela- tively easy to discriminate between thermal and nonthermal features.

\subsection{Spatially Resolved Synchrotron Emission}

AE Aquarii was also resolved in VLBI, showing an expanding source (at a speed of $\sim 0.01 \mathrm{c}$ ) on a half-hour timescale while the radio intensity decreased from a maximum (during a flare) to a minimum, until it reached a size of about four orbital radii (A. E. Neill, 1991, personal communication). This is again consistent with the model of expanding synchrotronemitting magnetized clouds.

\section{EVIDENCE OF TeV GAMMA-RAY EMISSION FROM AE Aqr}

$\mathrm{TeV} \gamma$-rays $\left(E_{\gamma}>10^{12} \mathrm{eV}\right.$ ) from AE Aquarii have been reported by two independent groups (Bowden et al. 1992; Meintjes et al. 1992). If these reports are correct, they would also require efficient particle accelerators near the white dwarf.

\subsection{The Potchefstroom (S.A.) Detections}

Meintjes et al. (1992) have shown that the detected signal has the same temporal behavior as observed in optical during flares. The $\mathrm{TeV}$ radiation was detectable at the spin frequency $F_{0}$ and at a frequency which is slightly redshifted from $F_{0}$ after all the available observations between 1988 and 1991 were added together. This behavior was independently confirmed after analysing the 1992 data (Meintjes 1993). The significance is about $4 \sigma$. The strongest individual detection is shown in Figure 3, and the signal is clearly above the background. The frequency is redshifted by $0.7 \%$ relative to $F_{0}$; similar shifts are seen in the optical as shown in Figure $2 b$.

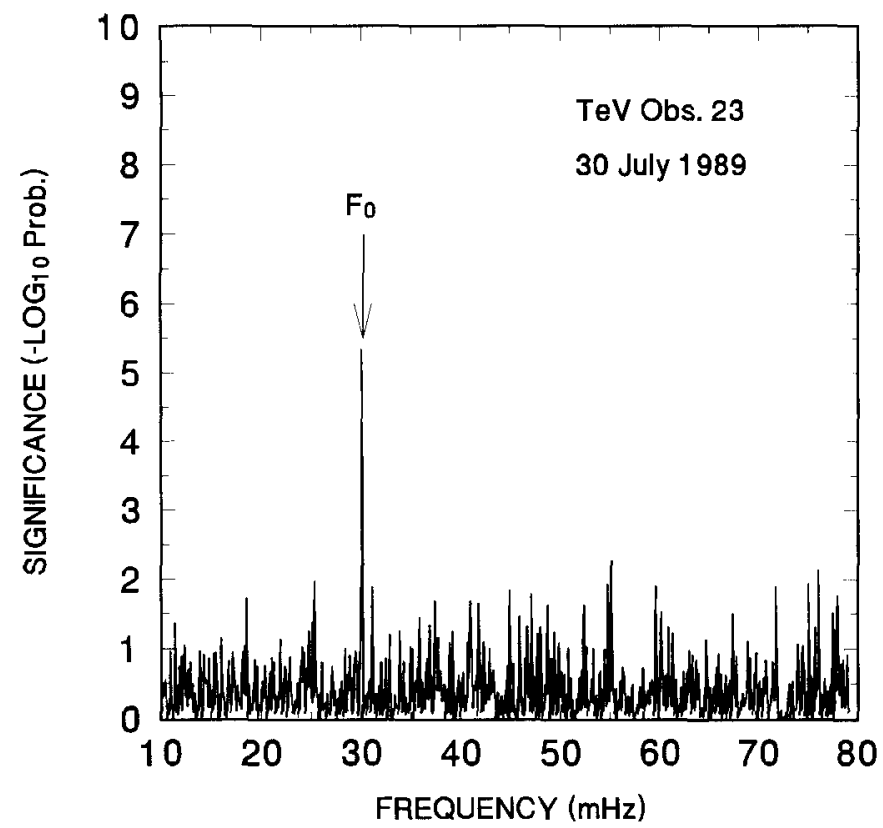

FIg. 3.-Power spectrum of the Potcefstroom TeV observation no. 23 of 1989 July 30 . The peak on the power spectrum is redshifted by $\sim 1 \%$ relative to $F_{0}$ (reproduced from Meintjes et al. 1992.) 

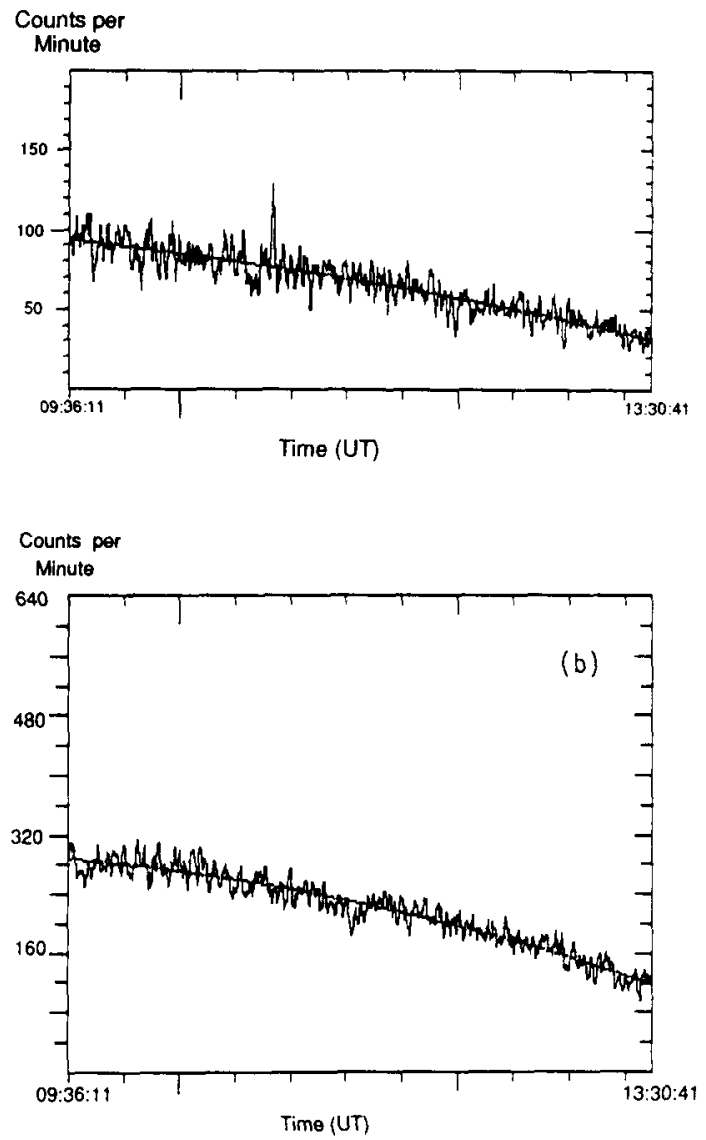

Fig. 4.-Summed count rate of AE Aqr on 1990 October 13 from both telescopes of Bowden et al. (1992) at $\gamma$-ray energies above $350 \mathrm{GeV}$. In $(a)$ the ON-source channel is displayed showing a short burst significant at the $6 \sigma$ level, whereas the OFF-source channel is displayed in $(b)$ (reproduced from Bowden et al. 1992.)

\subsection{The Narrabri (N.S.W.) Detections}

Bowden et al. (1992) reported the simultaneous detection of a $6 \sigma$ burst in TeV $\gamma$-rays by two independent telescopes on 1990 October 13 (the combined count rate is shown in Fig. 4.) The fact that the burst was also pulsed with a period of $16.5 \mathrm{~s}$ (i.e., half the spin period of the white dwarf), and given an observed photon spectrum which is significantly (at the $3 \sigma$ level) flatter than the cosmic-ray noise spectrum, adds to the significance of this detection. It was clearly shown that this burst was due to real $\gamma$-rays and not due to instrumental effects.

Following this report, an optical burst with similar features as seen in TeV (i.e., structure, duration, and pulsed fraction) was discovered by De Jager \& Meintjes (1993).

\section{CONVERSION EFFICIENCY OF ACCRETION POWER TO ACCELERATED PARTICLES}

$A E$ Aqr is one of the most efficient converters of accretion power to power in accelerated particles when considering accreting white dwarfs and neutron stars. This evidence follows from both radio and $\gamma$-ray observations:
The peak synchrotron luminosity is $S_{\nu} \sim 100 \mathrm{mJy}$ at $\Delta \nu \sim$ $\nu=250 \mathrm{GHz}$, implying a luminosity of at least $2 \times 10^{29} \mathrm{ergs}$ $\mathrm{s}^{-1}$. This implies a ratio of synchrotron to accretion power of

$$
\eta=\frac{4 \pi d^{2} S_{\nu} \Delta \nu}{G M M / R}
$$

which equals 0.002 if the radio emission is generated during optical quiescence, but $\eta \sim 10^{-4}$ during the optical flaring state. To put these numbers into perspective we can compare this value with $\eta \sim 6 \times 10^{-4}$ as obtained for an accreting source like Cyg X-3 when it experiences one of its gaint radio flares. However, in the case of AE Aqr these flares occur on a timescale of hours, whereas for Cyg X-3 the repetition timescale is about once every 6 months (Johnston et al. 1986.) Thus, the time averaged conversion efficiency for AE Aqr exceeds that of Cyg X-3 significantly. Since most of the electron energy is lost to adiabatic expansion, the conversion efficiency of accretion power to relativistic electrons would also be significantly larger than the above-mentioned values of $\eta$. One can also calculate the ratio of adiabatic relative to synchrotron losses, which, when multiplied with the synchrotron luminosity, gives an estimate of the total power in electrons. It can be shown that this ratio is of the order of hundreds, so that a significant fraction of the accretion luminosity must be converted into relativstic particles. However, the largest uncertainty in this calculation is the initial source radius, so that reliable estimates cannot be made at this stage.

A relatively high efficiency is also required for the reported $\mathrm{TeV} \gamma$-ray emission, since the time averaged luminosity $L_{\gamma} \sim$ $2 \times 10^{32} \mathrm{ergs} \mathrm{s}^{-1}$ (Meintjes et al. 1992), but may be as high as $10^{33} \mathrm{ergs} \mathrm{s}^{-1}$ during a large burst (Bowden et al. 1992).

\section{PULSAR-LIKE SPINDOWN}

A high-luminosity source is needed to provide the relativistic particles. Relativistic electrons are needed to explain the radio emission, whereas very high energy protons or electrons would be needed to explain the claimed $\mathrm{TeV} \gamma$-ray signals. In this discussion only the necessary potential drop and luminosity will be calculated from fundamental principles given the unique spindown.

An analysis of all the available optical pulse timings between 1978 and 1992 (De Jager et al. 1993) have shown the existence of a period derivative of $5.6 \times 10^{-14} \mathrm{~s} / \mathrm{s}$, which would result in a spindown power of

$$
I \Omega \dot{\Omega}=6.3 \times 10^{33} I_{50} \operatorname{ergs~s}^{-1}, \sim 60 \frac{G M \dot{M}_{q}}{R},
$$

where $\dot{M}_{q}$ indicates the accretion rate during quiescence. Such a situation was originally considered by Priedhorsky (1986) for millisecond pulsars in LMXB, which may explain the nonthermal radio emission found in, for example, Sco X-1. During the times of low accretion rate, the pulsar spins down, and energy is extracted from the flywheel which results in nonthermal radio emission. This case was also considered by Treves \& Bocci ( 1987) in a flywheel model for Cyg X-3 where the power for accelerating particles comes again from a radio pulsar-type spindown, which is achieved during a time of low 
accretion. In this case we do not see the spindown power in optical, but this power is spent in accelerating particles, stellar wind formation, as well as magnetic dipole radiation. The reasoning for such a type of spindown in AE Aqr is the following:

It can be shown that the large period derivative and moderate accretion rate are clearly incompatible with each other in the Ghosh \& Lamb (1979) model for disk-fed compact objects. There is no solution for their equations, so that the disk inner edge radius $r_{i}$ is outside the corotation radius $r_{c}$, during which no accretion is possible due to the propeller effect. One can also calculate $r_{i}$ from the magnetic moment and accretion rate, and the same conclusion (i.e., that $r_{i}>r_{c}$ ) is reached. Furthermore, the presence of QPO features which are redshifted relative to the spin frequency is also consistent with Keplerian motion just outside the corotation radius $r_{c}$. Another peculiar aspect is that $\mathrm{AE} \mathrm{Aqr}$ is the only cataclysmic variable which does not show flickering during quiescence (Bruch 1991). If flickering is also associated with accretion onto the white dwarf, then this observation also indicates that little or no accretion is taking place during quiescence. This situation (absence of accretion) was first studied by Shvartsman (1970). Davies \& Pringle (1981) have investigated this for spherical accretion. In the context of accretion disks, the so-called "dead" or "accumulator" disks have been studied by Syunyaev \& Shakura (1977). In their model for low accretion rates, angular momentum extracted from the compact object flows outwards over the disk from the inner edge. This is accompanied by energy liberated in the disk, so that all the $I \Omega \dot{\Omega}$ losses can be accounted for. However, this is not the case for AE Aqr: we know that AE Aqr has the lowest accretion rate of the known DQ Her-type objects, which may be the result of a hibernation process (which is common in cataclysmic variables), that is, it was spun up to a minimum period at a previous epoch (high $\dot{M}$ ), but $\dot{M}$ is relatively low at the present epoch and the white dwarf is spinning down due to the lack of an accretion torque. AE Aqr does not fit in the picture of Syunyaev \& Shakura (1977) since the spindown power is significantly larger than the observed disk + white dwarf luminosity.

Our proposed model is consistent with the idea of Michel \& Dessler ( 1981 ) for "dead disks" around pulsars, which came as an answer to the basic problem of current closure in pulsars: all conventional pulsar models (which are derived from the classic Goldreich-Julian model) fail to provide a return current for the outflowing pulsar current from the polar caps, whereas Michel \& Dessler ( 1981 ) proposed the existence of fossil collapse disks around active pulsars which can easily explain how the circuit closes. In the general framework of Michel \& Dessler (1981) and Michel (1983), the wind zone is defined by those field lines originating on the polar cap, but which are expelled by the disk due to the large $\boldsymbol{j} \times \boldsymbol{B}$ forces, whereas the auroral zone is defined by those field lines which connects the star with the disk. This zone would also correspond more or less in position to the accretion curtain as discussed for the pulsed X-rays in $\S 1$, except that the auroral zone is supposed to maintain its properties in the absence of significant accretion. The net charge density above the polar caps is expected to be close to the Goldreich-Julian density in general, even if a significant amount of particles have been pulled from the disk into the stellar magnetosphere by electric fields (Hamilton, Lamb, \& Miller 1993). For AE Aqr we find

$$
n_{\mathrm{GJ}}=\frac{\Omega B_{s}}{2 \pi e c} \sim 2 \times 10^{3} \mathrm{~cm}^{-3}
$$

given a typical surface field strength of $B_{s}=10^{6} \mathrm{G}$. The polar cap area is estimated assuming that the field line from white dwarf (with radius $R$ ) to the inner edge of the disk is approximately dipolar:

$$
A_{\mathrm{pc}}=\frac{1.6 \pi R^{3}}{r_{c}}=4 \times 10^{17}\left(\frac{R}{5 \times 10^{8} \mathrm{~cm}}\right)^{3} \mathrm{~cm}^{2}
$$

For the standard radio pulsar (diskless) models, the corotation radius is replaced with the light cylinder radius, leading to much smaller polar cap areas. The total current into (or out of) the polar caps follows from the assumption of having the Goldreich-Julian density above the polar caps. The magnitude of the current, counting both polar caps, is

$$
I_{0}=\frac{1.6 \mu \Omega}{r_{c}} \sim 2 \times 10^{22} \mu_{32} \text { statamp }
$$

and when calculating the magnetic flux into the wind zone for each pole, we obtain the stellar wind torque on the star and hence the total spindown power, which is given by

$$
I \Omega \dot{\Omega}=\frac{0.64 \mu^{2} \Omega^{2}}{c r_{c}^{2}} .
$$

This power is lost in the form of a relativistic stellar wind, which may explain the observed radio synchrotron emission as observed well outside the binary orbit (see $\S 2.3$ ). The above equation can be set equal to the observed spindown power if the magnetic moment $\mu=1.3 \times 10^{32} \mathrm{G} \mathrm{cm}^{3}$, which is quite reasonable for DQ Her type CVs: one can check for consistency by using this value of $\mu$ to calculate the disk inner edge radius from Ghosh \& Lamb (1991) giving

$$
r_{i} \sim 0.5\left(\frac{\mu^{4} G M}{L^{2} R^{2}}\right)^{1 / 7}=5 r_{c} L_{33}^{-2 / 7}
$$

This radius is indeed larger than the corotation radius given the accretion rate $L_{33}=L_{\text {acc }} / 10^{33}$ ergs $\mathrm{s}^{-1} \sim 1$, and would be consistent with the observation of quasi periodic oscillations at Keplerian frequencies corresponding to $r_{i}>r_{c}$. As matter accumulates at $r_{i}$ due to continuous accretion from the secondary star, $r_{i} \rightarrow r_{c}$, giving the redshifted Keplerian QPO features close to the spin frequency as shown in Figure $2 b$. This would be coincident with flare-like activity in the optical, triggered by the "magnetospheric gating" effect as suggested by Van Paradijs et al. (1989).

The current flow results in particles of one sign flowing out of the polar cap into the wind zone, and the electric field present can pull particles of the opposite sign toward the stellar surface. If the potential drop is large enough, the returning particles can penetrate deeply into the stellar surface, heating 
each polar cap, resulting in blackbody emission from both polar caps. This would be consistent with the observed optical double pulse with a blackbody spectrum. The current out of the polar caps returns through the disk toward the star and requires a current density (Michel 1983)

$$
J=\frac{I_{0}}{2 \pi R d}\left(\frac{r_{c}}{R}\right)^{1 / 2},
$$

where $d$ is the width of the current sheet. In the terminology of Michel (1983) this sheet is called the "auroral zone" which connects the disk inner edge with the stellar surface, and with this return current, the circuit is closed. Rewriting the luminosity of the auroral zone (Michel 1983) in terms of the spindown power gives

$$
L_{\mathrm{az}}=\frac{2.5 d}{R}\left(\frac{r_{c}}{R}\right)^{1 / 2} I \Omega \dot{\Omega} \ll I \Omega \dot{\Omega}
$$

The geometry of the auroral zone closely resembles the thin accretion curtain which explains the broad X-ray pulse profiles as discussed previously. Particles returning to the stellar surface via this auroral zone can also heat the stellar surface to provide the observed X-ray bremstrahlung (see Fig. 1), but in this case the energy of the particles must be much less than those in the wind zone (which resulted in the blackbody spectrum), since thermal bremstrahlung requires heating in or above the photosphere (heating below the photosphere would also result in a blackbody spectrum). ${ }^{1}$ Setting $L_{\mathrm{az}}$ equal to the $\mathrm{X}$-ray luminosity of a few times $10^{30} \mathrm{ergs} \mathrm{s}^{-1}$ (Patterson et al. 1980 ) requires the width $d \sim 10^{5} \mathrm{~cm}$ which is still much less than the stellar radius. In the model of Michel this width is determined by the electron conductivity, giving $d \sim 100 \mathrm{~cm}$ for white dwarfs if resistivity is determined by scattering of electrons on, say, partially stripped iron atoms.

\section{PARTICLE ACCELERATION}

The model of Michel ( 1983 ) considered the auroral zone as the site for particle acceleration to explain the radio and $\gamma$-ray emission from pulsars, but the accelerating potential and luminosity of this zone is much smaller than predicted by other conventional pulsar models (see, e.g., Harding 1981). Chanmugan \& Brecher (1985) investigated the star-disk dynamo where ultrarelativistic plasma jets are emitted from the disk along $\boldsymbol{B}$. In a different approach Cheng \& Ruderman (1991) considered the existence of vacuum gaps between the star and disk as the site for particle acceleration. However, the existence of vacuum gaps was questioned (Lamb, Hamilton, \& Miller 1993). The latter authors have shown that the large electric fields would pull particles from the disk, resulting in a quench-

\footnotetext{
I A similar situation was proposed for Geminga, where heating deep inside the polar cap by reversed high-energy particles gives rise to a soft blackbody oscillation spectrum, but the less penetrating gamma rays are absorbed in the photosphere to give a harder thermal bremstrahlung spectrum (Harding, Ozernoy, \& Usov 1993). If gamma rays are emitted in AE Aqr by any returning particle, then such radiation would also be reprocessed into X-ray thermal bremstrahlung in the photosphere, which would contribute to the observed X-rays.
}

ing of any vacuum gap. The current flow in the model of Lamb, Hamilton \& Miller (1993) is similar to the flow proposed by Michel (1983), except that their general description extends to the case where accretion is significant. Their analysis involves the calculation of the EMF

$$
\mathrm{EMF}=\oint\left(E+\frac{1}{c} v \times B\right) \cdot d \ell
$$

along a closed circuit connecting the same polar cap region as suggested by Michel (1983) (as used in the previous section to calculate the spindown power) with the disk via poloidal field lines. A similar integration was performed by Brink et al. (1990) and Cheng \& Ruderman (1991). This gives two terms, depending on whether the star or disk dominates the contribution to the EMF. For AE Aqr with $r_{i}>r_{c}$ in quiesence we expect the stellar term to dominate, giving an EMF or potential drop of

$$
\Delta V=\frac{\mu \Omega}{2 c r_{i}}=6 \times 10^{13} \mu_{32}\left(\frac{r_{c}}{r_{i}}\right) \mathrm{V}
$$

If we take $r_{i}=c / \Omega$, that is, at the light cylinder radius, then we recover the classic potential drop across the polar cap for isolated radio pulsars, but with $r_{i} \ll c / \Omega$, a much smaller field strength is needed to provide a large potential drop and significant spindown as originally proposed by Michel (1983).

Since the resistance of the circuit is very small (Lamb et al. 1993), we expect large conduction currents to be driven by this EMF along a similar current pattern as proposed by Michel (1983) (see the previous section). The important analysis made by Lamb et al. ( 1993) and Hamilton et al. (1993) is that instead of "shorting out" the EMF and thereby preventing particle acceleration as some authors have argued (see, e.g., Wang 1986; Katz \& Smith 1988; Mitra 1991), the low internal resistance of the disk-star generator circuit allows the conduction currents to become large enough to trigger processes that accelerate particles. This follows when considering the combined inductance and resistance of the circuit, which allows the current to grow, leading to microscopic and/or MHD instabilities before the current has saturated to a steady state of EMF/resistance.

When extending the instability analysis to AE Aqr (as done by Hamilton et al. 1993), we find that micro-instabilities would occur in the most tenuous regions of the magnetosphere where the current density exceeds

$$
j_{\text {micro }}=e n_{e}\left(k T_{e} / m_{e}\right)^{1 / 2} \sim 10^{8} n_{9} T_{6}^{1 / 2},
$$

where $m_{e}$ is the electron mass, $n_{e}=n_{9} 10^{9} \mathrm{~cm}^{-3}$ is the electron density, and $T_{e}=T_{6} \times 10^{6} \mathrm{~K}$ is the corresponding temperature. Comparing the return current from equation (11) with $j_{\text {micro }}$ shows that the current sheet has to be thin:

$$
d<2 \times 10^{5} n_{9}^{-1} T_{6}^{1 / 2} \mu_{32} \mathrm{~cm}
$$

with $n_{9}$ as the major uncertainty in this condition: the presence of a disk implies that $n_{e}$ in even the most tenuous parts of the 
magnetosphere may greatly exceed the Goldreich-Julian density $n_{\mathrm{GJ}} \sim 10^{3} \mathrm{~cm}^{-3}$, although the net charge density is still expected to be $\left(n_{+}-n_{-}\right) \sim n_{\mathrm{GJ}}$. In fact, if full accretion takes place, the electron density may easily exceed $10^{12} \mathrm{~cm}^{-3}$, leading to the constraint of having $d<100 \mathrm{~cm}$ for a micro-instability to occur in the auroral zone. It is thus clear that this condition may only rarely be met, but if accretion is somehow inhibited by the propeller effect (i.e., when the system is in optical quiescence), $n$ is expected to be much lower which relaxes the constraint on $d$, leading to micro-instabilities and possibly double layer formation before magnetic reconnection takes place (Spicer 1982; Hamilton et al. 1993). Double layer formation involves charge separation maintained by a balance between inertial and electrostatic forces, leading to a localized electric field over a length $\ell$, over which ions and/or electrons are accelerated to monoenergetic values of the order $e E \ell=$ $e V_{\mathrm{DL}}$. If the relativistic double layer is bordered by a thermal plasma consisting of ions with mass $m_{i}$ and electrons, we find that

$$
V_{\mathrm{DL}} \sim\left(\pi m_{i} c j / e\right)^{1 / 2} \ell=10^{13} \ell_{8} n_{9}^{1 / 2} T_{6}^{1 / 4} \mathrm{~V}
$$

for a current density equal to $j_{\text {micro }}$ (Hamilton et al. 1993) in the presence of thermalized helium ions.

Thus, acceleration to $\mathrm{TeV}$ energies is possible in $\mathrm{AE}$ Aqr, but is expected to be a rare event since the current density may only rarely exceed $j_{\text {micro }}$. The $\mathrm{TeV}$ event would also be flarelike, since the double layer itself is unstable. The slower rotating disk at $r_{i}>r_{c}$ would lead to the creation of azimuthal field $\left(B_{\phi}\right)$ and current $\left(j_{\phi}\right)$ components, hence particle acceleration would also take place in a frame which is rotating slightly slower than the star, resulting in the observation of redshifted $\mathrm{TeV}$ signals as shown in Figure 3.

In most cases we expect the threshold for micro-instabilities not to be met. In this case magnetic reconnection is more likely to occur due to the presence of higher electron densities and the continuously growing $B_{\phi}$ component (Parker 1979; Hamilton et al. 1993), leading to plasma heating and bulk acceleration to the Alfvén velocity $v_{\mathrm{A}}$ which would be consistent with the observation of expanding $\left(v_{\mathrm{A}} \sim 0.01 \mathrm{c}\right)$ synchrotron emitting (by mildly relativistic electrons) clouds if

$$
B \sim 430 n_{9}^{1 / 2} \mathrm{G} .
$$

(See Aly \& Kuipers 1990 for a detailed discussion on flare-like reconnection events in accretion disks leading to particle acceleration.) This mechanism would account for the ever-present radio emission, since the constraint on $n$ is much less severe in this case. In fact, from the model of BDC a typical radio flare would require $n_{9} \sim 1$ and $B$ of the order of a few hundred Gauss, which is consistent with equation (18), and radio emission is expected to occur at distances of a few times $r_{c}$ from the white dwarf where such field strengths are expected.

\section{CONCLUSION}

AE Aquarii contains the most rapidly spinning white dwarf known to us, and with the spindown power exceeding the accretion luminosity, we have pulsar-like properties in this unique system. The spindown power can be accounted for if a relativistic wind is ejected by the polar caps. The large EMF set up by the fast rotating white dwarf drives a conduction current flowing out of the polar caps and returning to the star via the accretion disk (which may be slowly or nonaccreting during optical quiescence). These two different sites on the stellar surface (i.e., where the current exits and returns) provides the potential sites for the optical and X-ray oscillations which are known to have different pulse profiles and spectra.

It was shown that it is relatively easy to produce radio synchotron emission via reconnection events, leading to the everpresent flare-like radio synchrotron emission, although the details have not been worked out. If the current density is large enough in a tenuous magnetosphere, double layers can form leading to large electric fields and hence mono-energetic electrons and ions with energies exceeding a TeV. This suggests that the TeV should be produced when the system is in absolute quiescence so that the magnetosphere can be tenuous enough for a double layer to be formed when driven by a large "pulsar" type current resulting from the rapid spindown. Simultaneous optical/ TeV observations would be necessary to confirm this.

Accretion from the secondary star is expected to be a continuous process, and if accretion is inhibited during quiescence ( since $r_{i}>r_{c}$ ), the ram pressure at $r_{i}$ would increase until the disk inner edge is pushed inside the corotation radius, leading to uninhibited accretion and the triggering of optical flares as seen quite regularly in AE Aqr (see the "magnetospheric gating" effect as proposed by van Paradijs et al. 1989 for AE Aqr). If the model for $\mathrm{TeV}$ acceleration within double layers holds, we would expect a cessation of $\mathrm{TeV}$ production during optical flares, since the magnetosphere would be too thick for the formation of double layers.

The author would like to thank Piet Meintjes and Bill Welsh for useful discussions.

\section{REFERENCES}

Abada-Simon, M., et al. 1993, ApJ, in press

Aly, J. J., \& Kuipers, J. 1990, A\&A, 227, 473

Bastian, T. S., Dulk, G. A., \& Chanmugan, G. 1988, ApJ, 324, 431 (BDC)

Berriman, G., et al. 1981, MNRAS, 217, 327

Bookbinder, J. A., \& Lamb, D. Q. 1987, ApJ, 323, L131

Bowden, C. C. G., et al. 1992, Ap. Phys. 1, 47

Brink, C., et al. 1990, in Proc. 21 st Internat. Cosmic Ray Conf. (Adelaide), 2,283

Bruch, A. 1991, A\&A, 251, 59

Chanmugan, G., \& Brecher, K. 1985, Nature, 313, 767

Cheng, K. S., \& Ruderman, M. A. 1991, ApJ, 373, 187

Davies, R. E., \& Pringle, J. E. 1981, MNRAS, 196, 209
De Jager, O. C., \& Meintjes, P. J. 1993, A\&A, 268, L1

De Jager, O. C., et al. 1993, MNRAS, submitted

Eracleous, M., et al. 1991, ApJ, 382, 290

Ghosh, P., \& Lamb, F. K. 1979, ApJ, 234, 296

. 1991, in Neutron Stars: Theory and Observation, ed. J. Ventura \& D. Pines (NATO ASI Series C), 344, 363

Hamilton, R. J., Lamb, F. K., \& Miller, M. C. 1993, in Proc. 1992 Compton Gamma-Ray Observatory Symposium, ed. N. Gehrels (New York: AIP), in press

Harding, A. K. 1981, ApJ, 245, 267

Harding, A. K., Ozernoi, L. M., \& Usov, V. V. 1993, MNRAS, in press Johnston, K., et al. 1986, ApJ, 309, 707 
Katz, J., \& Smith, I. A. 1988, ApJ, 326, 733

Lamb, D. Q. 1988, in Polarized Radiation of Circumstellar Origin, ed. G. V. Coyne et al. (Vatican Observatory, Vatican City State)

Lamb, F. K., Hamilton, R. J., \& Miller, M. C. 1993, Proc. 1992 Compton Gamma-Ray Observatory Symposium, ed. N. Gehrels (New York: AIP), in press

Meintjes, P. J. 1993, Ph.D. thesis, Potchefstroom Univ.

Meintjes, P. J., et al. 1992, ApJ, 401, 325

Michel, F. C. 1982, Rev. Mod. Phys., 54, 1 1983, ApJ, 266, 188

Michel, F. C., \& Dessler, A. J. 1981, ApJ, 251, 654

Mitra, A. 1991, ApJ, 370, 345

Paczyński, B. 1990, ApJ, 365, L9

Parker, E. N. 1979, Cosmical Magnetic Fields (Clarendon: Oxford)
Patterson, J. 1979, ApJ, 234, 978

Patterson, J., et al., 1980, ApJ, 240, L133

Priedhorsky, W. 1986, ApJ, 306, L97

Shvartsman, V. F. 1970, Radiofizika, 13, 1852

Spicer, D. S. 1982, Space Sci. Rev., 31, 351

Syunyaev, R. A., \& Shakura, N. I. 1977, Pis'ma Astron., 3, 216

Szkody, P. 1977, ApJ, 217, 140

Tanzi, E. G., et al. 1981, PASP, 93, 68

Treves, A., \& Bocci, F. 1987, MNRAS, 225, 39P

Van Paradijs, J., et al. 1989, A\&A, 79, 205

Wang, Y. M. 1986, Ap\&SS, 121, 193

Warner, B., \& Wickramasinghe, D. T. 1991, MNRAS, 248, 370

Welsh, W. F., Horne, K., \& Gomer, R. 1993, ApJ, 410, L39

Welsh, W. F., Horne, K., \& Oke, R. 1993, ApJ, 406, 229 